Bachrach, Phys. Rev. Lett. 42, 104 (1979).

${ }^{9}$ P. A. Lee and G. Beni, Phys. Rev。 B 15, 2862 (1977).

${ }^{10}$ P. H. Citrin, P. Eisenberger, and B. M. Kincaid, Phys. Rev. Lett. 36, 1346 (1976).

${ }^{11}$ H. Petersen and C. Kunz, Phys. Rev. Lett. $\underline{35}, 863$ (1975).

${ }^{12}$ Because of the symmetry of the initial states, the photon polarization does not play a major role in the analysis of our experimental data.

${ }^{13}$ Experiments have also been carried out for the $j=\frac{3}{2}$ component of In $4 d$ and of Sn $4 d$. The intensity modulations discussed here were similar for these peaks and for their $j=\frac{5}{2}$ partners shown in Figs. 1 and 2. Differences between the $j=\frac{5}{2}$ and the $j=\frac{3}{2}$ intensity versus energy curves have been found to exist for the atomic background to which the modulations are superimposed and they will be discussed elsewhere.

\author{
${ }^{14}$ G. Margaritondo, J. E. Rowe, and S. B. Christman, \\ Phys. Rev. B 19, 2850 (1979). \\ ${ }^{15} \mathrm{U}$. Fano and J. W. Cooper, Rev. Mod. Phys. $\underline{40}, 441$ \\ (1968). \\ ${ }^{16}$ N. G. Stoffel and G. Margaritondo, unpublished. \\ ${ }^{17}$ R. W. G. Wyckoff, Crystal Structures (Interscience, \\ New York, 1964), Vol. 2. \\ ${ }^{18}$ A. P. Lambros, D. Gerales, and N. A. Economou, \\ J. Phys. Chem. Solids 35, 537 (1974). \\ ${ }^{19} \mathrm{D}$. Gregory and M. Fink, At. Data Nucl. Data Tables \\ 14,39 (1974). Because of the slow variation of the \\ phase shift with the atomic number, in our estimates \\ we have employed $\mathrm{Cd}(Z=48)$ instead of In $(Z=49)$ and \\ $\mathrm{Sn}(Z=50), \mathrm{Br}(Z=35)$ instead of Se $(Z=34)$, and $\mathrm{P}(Z$ \\ 15) instead of $\mathrm{S}(Z=16)$. The phase shift for the $180^{\circ}$ \\ scattering at the neighboring atom has been approxi- \\ mated with the calculated value for $178^{\circ}$ scattering.
}

\title{
Photoacoustic Effect in Solids
}

\author{
C. L. Cesar, H. Vargas, J. A. Meyer, and L. C. M. Miranda \\ Instituto de Física, Universidade Estadual de Campinas, 13100 Campinas, São Paulo, Brazil
} (Received 6 December 1978)

\begin{abstract}
A quantitative derivation is presented for the production of the acoustic signal in a photoacoustic cell, taking into account the finite surface thermal resistance of the solid.
\end{abstract}

The photoacoustic (PA) spectroscopy has proved $^{1-6}$ to be an extremely useful tool for studying absorption spectra of crystalline, powdered, and amorphous solids as well as biological materials and liquids. In this Letter we develop the theory for the PA signal of a solid, taking into account, however, the surface thermal resistance of the sample. Our approach follows closely that of Rosencwaig and Gersho ${ }^{2}$ (RG) and we refer to their work for further details.

Consider a simple cylindrical cell of diameter $D$ and length $L=l+l_{b}+l_{g}$. The sample is considered to be in the form of a disk having diameter $D$ and length $l$. A sinusoidally chopped monochromatic light with wavelength $\lambda$ is incident on the solid with intensity $I=I_{0}(1+\cos \omega t) / 2$, where $I_{0}$ is the incident monochromatic light flux and $\omega$ is the chopping frequency. We further assume that the gas and the backing materials are not light absorbing. We define the following parameters: $k_{i}$, thermal conductivity of material $i ; c_{i}$, specific heat of material $i ; \rho_{i}$, density of material $i ; \alpha_{i}=k_{i} / \rho_{i} c_{i}$, thermal diffusivity of material $i ; a_{i}=\left(\omega / 2 \alpha_{i}\right)^{1 / 2}$, thermal diffusion coefficient of material $i ; \mu_{i}=1 / a_{i}$, thermal diffusion length of material $i ; \beta$, optical absorption coefficient of the solid sample. Here, the subscripts $i=s, g$, or $b$, denote the sample, gas, or backing mater- ial, respectively. Let $\varphi_{i}(x, t)$ denote the temperature in material $i$ relative to ambient temperature $\left(T_{0}\right)$ due to the light into heat conversion process. By neglecting the heat losses by radiation at the lateral surfaces (the inclusion of linear heat losses can easily be done), the temperature in cell obeys the same thermal diffusion equations as those of RG theory. ${ }^{2}$

The real part of $\varphi_{g}(x, t)$ is, of course, the solution of physical interest. This, in turn, is obtained by solving the thermal diffusion equations together with the appropriate boundary conditions. In the RG (Ref. 2) and other theories ${ }^{3-5}$ the boundary conditions are temperature and heat-flux continuity at the sample boundaries $x=0$ and $x=-l$, together with the constraint that the temperature at the cell walls is at ambient temperature [i.e., $\left.\varphi_{g}\left(x=l_{g}, t\right)=\varphi_{b}\left(x=-l-l_{b}, t\right)=0\right]$. This latter constraint is a reasonable assumption for metallic cell walls. On the other hand, the condition of temperature continuity at the faces $x=0$ and $x=-l$ is a very restricting one. In the general case, temperature continuity at the face of two bodies is only valid for very intimate contact, such as a soldered joint. 6 In all other cases, even for optically flat surfaces pressed lightly together, heat transfer between two media takes place largely by the linear heat-transfer mechanism, 
in which case the heat flow across the boundary of two media $i$ and $j$ is proportional to the temperature difference between them, i.e., $H\left(\varphi_{i}-\varphi_{j}\right)$. Here $H$ is a constant called ${ }^{6}$ the coefficient of heat transfer. The constant $H$ is often specified in terms of the surface thermal resistance $R=1 / H$, and it depends on the actual heat-loss mechanism. Hence, for nonvanishing surface thermal resistance, the temperature continuity condition at the boundary of two media $i$ and $j$ should be replaced by the requirement that ${ }^{6}$

$$
-K_{i} \partial \varphi_{i} / \partial n=H_{i}\left(\varphi_{i}-\varphi_{j}\right),
$$

where $\delta / \partial n$ denotes differentiation in the direction of the outward normal to the boundary face of material $i$. We note that in the case of zero ther- mal resistance (i.e., $H_{i}=\infty$ ), Eq. (1) reduces to the temperature continuity condition. Physically, this case of zero thermal resistance means that the heat transfer between the two media is instantaneous or, alternatively, that the surface emissivity is infinite. Since the heat-transfer time is roughly of the order of the thermal diffusion time in the sample $\left(\sim l^{2} / \alpha_{s}\right)$, one should therefore abandon the temperature continuity condition at the sample boundaries. Neglecting transients as well as the dc components, the solution $\varphi_{g}(x, t)$ employing the new boundary conditions is then given by

$$
\varphi_{g}(x, t)=\theta \exp \left(-\sigma_{g} x\right) e^{j \omega t},
$$

where

$$
\theta=\frac{\beta I_{0}}{k_{s}\left(\beta^{2}-\sigma_{s}^{2}\right)}\left(\frac{(r-1)(\zeta+1) \exp \left(\sigma_{s} l\right)-(r+1)(\zeta-1) \exp \left(-\sigma_{s} l\right)-2(r-\zeta) e^{-\beta l}}{(\zeta+1)(g / h+1+g) \exp \left(\sigma_{s} l\right)+(\zeta-1)(g / h+1-g) \exp \left(-\sigma_{s} l\right)}\right) .
$$

Here $g=k_{g} \sigma_{g} / k_{s} \sigma_{s}, \quad b=k_{b} \sigma_{b} / k_{s} \sigma_{s}, r=\beta / \sigma_{s}, h=H_{s} /$ $k_{s} \sigma_{s}$, and $\zeta=b h /(b+h)$. In the limit of zero thermal resistance (i.e., $h \rightarrow \infty$ ) Eq. (3) reduces to the result of the RG theory. ${ }^{2}$

Once we know the temperature in the gas, we now assume the RG acoustic piston model $^{2}$ for the production of the acoustic signal in the gas. By evaluating the spatially averaged temperature of the gas within the boundary layer, and proceeding similarly as in Ref. 2 , the incremental pressure, $\delta P(t)$, in the gas can then be written as

$$
\begin{aligned}
& \delta P(t)=\gamma P_{0} \delta x(t) / l_{g}=Q \exp [j(\omega t-\pi / 4)], \\
& Q=\gamma P_{0} \theta / \sqrt{2} l_{g} a_{g} T_{0},
\end{aligned}
$$

with $P_{0}$ being the ambient pressure and $\gamma$ is the ratio between the specific heats of the gas. Equation (4) is the expression for the PA signal one wants to discuss. As the expression for $Q$ is, in the general case, quite complicated we shall consider only a few special cases where it becomes relatively simple.

Optically opaque and thermally thin solid. - In this case the thermal diffusion length $\mu_{s}=a_{s}^{-1}$ is much larger than the solid thickness which, in turn, is much larger than the optical absorption length $\beta^{-1}$. I.e., one has $\beta l \gg 1$ and $\beta \gg a_{s}$. It is important to notice that this condition of optically opaque and thermally thin solid is not uniquely defined by the condition of a very black absorber such as carbon black; it does depend on both the sample length as well as on the working frequency range. We shall find it useful to rewrite the con- dition $l a_{s} \ll 1$ as

$$
\left(f \tau_{d}\right)^{1 / 2} \ll 1, \quad \tau_{d}=\pi l^{2} / \alpha_{s} .
$$

Here $\tau_{d}$ is the thermal diffusion time ${ }^{6}$ in the sample. Hence, by thermally thin solid we mean the situation in which the thermal diffusion time is much smaller than the chopping period. Setting in $\mathrm{Eq}$. (3) $\exp (-\beta l) \simeq 0, \exp \left( \pm \sigma_{s} l\right) 1$, and $|r| \gg 1$, assuming that $H_{s}$ is given by the blackbody mechanism ${ }^{6}$ (i.e., $H_{s}=4 \sigma e_{s} T_{0}^{3}$ with $\sigma$ being the StefanBoltzmann constant), and using the values of the physical parameters for typical gases and backings, one can show that $h \ll g \ll b$, so that $\zeta=h$ and $\theta \simeq(1-j) T_{0} / 4 k_{g} a_{g}$. We then get, from Eq. (4),

$$
Q=\frac{(1-j)}{2 \pi} \frac{\gamma P_{0} I_{0}}{2 \sqrt{2} l_{g} T_{0}} \frac{\alpha_{g}}{k_{g} f} .
$$

Equation (6) tells us that for optically opaque and thermally thin solids the PA signal is independent of $\beta$, varies inversely proportional to the frequency, and depends only on the thermal properties of the gas $\alpha_{g} / k_{g}$. The corresponding RG result differs from Eq. (6) only through its dependence on the thermal properties; instead of the factor $\alpha_{g} / k_{g}$, the RG theory predicts $\left(\alpha_{b} \alpha_{g}\right)^{1 / 2} / k_{b}$. I.e., the RG expression for the PA signal of an optically opaque and thermally thin solid depends on both the gas and the backing thermal properties, but has the same frequency dependence as ours $\left(f^{-1}\right)$.

Physically, the dependence on the thermal 
properties of the gas that we found can be understood as follows. The quantity $H_{s}$ measures the rate of heat transfer per unit area per unit time into the gas, so that one may define the length $l_{t}$ of heat transfer into the gas as $l_{t}=k_{s} / H_{s}=k_{s} R$, from which we can also define the corresponding time for heat transfer into the $\tau_{t}$, namely, $\tau_{t}$ $=\pi l_{t}{ }^{2} / \alpha_{s}$. We have

$$
l_{t} a_{s} \simeq h^{-1} \text {. }
$$

Since $h \ll 1$, it follows from Eqs. (7) and the condition $l a_{s} \ll 1$, that the length of heat transfer into the gas is much larger than the sample length. This, in turn, means that the heat generated within the very small surface layer $\left(\beta^{-1}\right)$ of the solid by the absorbed light is initially transferred into the gas instead of propagating first through the sample. In the case of zero thermal resistance, $l_{t}=0$, so that the heat in the surface layer of the solid first propagates through the sample. As a result the PA signal should now depend on the thermal properties of the backing.

Optically opaque and thermally thick solid. - This is the case where the thermal diffusion length $\mu_{s}$ is smaller than the sample length but larger than the optical penetration depth; i.e., one has $\beta l \gg 1, l a_{s} \gg 1$, and $\beta \gg a_{s}$. Hence, if we set in Eq. (3) $\exp (-\beta l) \simeq 0, \exp \left(-\sigma_{s} l\right) \simeq 0$, and $|r|$ $\gg 1$, one gets $\theta=-j I_{0} H_{s} / 4 k_{s} a_{s} k_{g} a_{g}$, which after substituting into Eq. (4) gives us

$$
Q=-\frac{j}{2 \pi^{3 / 2}} \frac{\gamma P_{0} I_{0} H_{s}}{2 \sqrt{2} l_{g} T_{0}} \frac{\alpha_{s}{ }^{1 / 2} \alpha_{g}}{k_{s} k_{g} f^{3 / 2}} .
$$

I.e., for optically opaque and thermally thick solids the acoustic signal is independent of $\beta$, varies inversely proportional to $f^{3 / 2}$, and depends on the thermal properties of both the sample and the gas. Its dependence on the thermal properties of the gas is the same as in the previous case of thermally thin solid. Actually, Eqs. (8) and (6) differ roughly by a factor $\left(l_{t} a_{s}\right)^{-1}$. The corresponding $\mathrm{RG}$ result differs from $\mathrm{Eq}$. (8) through its dependence on both the chopping frequency $\left(f^{-1}\right)$ as well as on the thermal properties of the gas.

Summarizing, the differences we have found for the frequency dependence of the PA signal between the cases of thermally thick $\left(\sim f^{-3 / 2}\right)$ and thermally thin $\left(\sim f^{-1}\right)$ samples may be understood as follows. As previously noted, the case of thermally thin sample means that the solidgas heat transfer is instantaneous. I.e., the time it takes for the heat generated at the sample be transferred to the gas is much smaller than the period $f^{-1}$. Hence, at a given light pulse, all the heat from the proceeding pulse has been completely transferred and the gas has thermalized. On the other hand, for the thermally thick case one has the opposite situation, namely, that a finite time is required for the solid-gas heat transfer. This means that the heat coming from a light pulse may still be being transferred at the moment of arrival of the next one. This makes these two cases physically different, hence producing a different time dependence, and one should expect a different frequency dependence. In the case of RG theory, however, the boundary condition used (temperature continuity) implies that the heat transfer is instantaneous for both cases. Consequently, it is no surprise that the frequency dependence $\left(\sim f^{-1}\right)$ found by $\mathrm{RG}$ should be the same for both thermally thin and thermally thick samples.

To compare the above predictions with those of $\mathrm{RG}$ theory we have performed measurements of the PA signal of a germanium sample of $480 \mu \mathrm{m}$ in thickness in a wide range of frequencies, changing, however, the cell's gas. Of course a more dramatic illustration of the differences brought in by the introduction of the surface thermal resistance would be to plot the frequence dependence of the PA signal for the thermally thick case. However, this kind of plot requires the knowledge of the cell's response (cell plus detection system) as a function of the chopping frequency. I.e., the experimentally observed PA signal is actually the product of Eq. (4) times the cell's response function. Since this is not an a priori known function, we avoided this difficulty by looking at the gas-parameter dependence of the PA signal. The gases used were helium and air. The ex-

TABLE I. Observed ratio of the PA signal of a Ge sample of thickness $l=480 \mu \mathrm{m}$ in the He-filled to that in the air-filled cell as a function of the chopping frequen-

\begin{tabular}{|c|c|}
\hline $\begin{array}{l}\text { Frequency } \\
\quad(\mathrm{Hz})\end{array}$ & $\frac{\left|Q_{\mathrm{Ge}}(\mathrm{He})\right|}{\left|Q_{\mathrm{Ge}}(\mathrm{air})\right|}$ \\
\hline 30 & $1.3 \pm 0.1$ \\
\hline 53 & $1.6 \pm 0.16$ \\
\hline 95.4 & $1.8 \pm 0.18$ \\
\hline 157 & $2.0 \pm 0.2$ \\
\hline 250 & $2.4 \pm 0.24$ \\
\hline 894 & $1.8 \pm 0.18$ \\
\hline 1530 & $1.3 \pm 0.13$ \\
\hline
\end{tabular}
cy. 


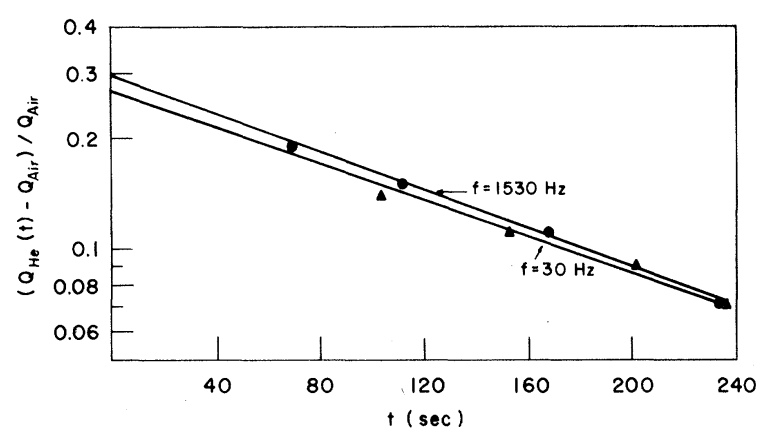

FIG. 1. Ratio of the PA signal of a Ge sample of 480 $\mu \mathrm{m}$ in thickness for an He-filled to an air-filled cell as a function of time. The circles and triangles are the experimental points for the chopping frequencies of 1530 and $30 \mathrm{~Hz}$, respectively.

perimental apparatus consisted primarily of a 500-W tungsten-filament lamp, a variable-speed light chopper, an aluminum cylindrical cell with an electrect microphone, and a lock-in amplifier. The light from the filament lamp with a filter to cut the contribution from wavelengths greater than $5000 \AA$. This assured us that the sample is optically opaque $\left(\beta \gtrsim 10^{4} \mathrm{~cm}^{-1}\right)$ for our light source. Using the appropriate values of the physical parameters for Ge $\left(\alpha_{s}=0.4 \mathrm{~cm}^{2} \mathrm{~s}^{-1} ; k_{s}=0.67\right.$

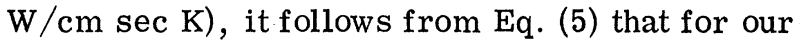
particular sample thickness, $\mathrm{Ge}$, is thermally thick for $f$ above $55 \mathrm{~Hz}$, and thermally thin below it. Hence, the ratio of the PA signal of our Ge sample for the helium-filled cell to that of the air-filled cell should give us the same value at both high and low frequencies. I.e., according to Eqs. (6) and (8) at both high frequencies (thermally thick) and low frequencies (thermally thin) one should get $\left|Q_{G e}(\mathrm{He})\right| / \mid Q_{G e}($ air $) \mid=1.3$. On the other hand, according to RG theory, this ratio would be equal to [cf. Eqs. (26) and (27) of Ref. 3] 2.7, both at high and low frequencies.

In Table I we present the ratio of the PA signal of our Ge sample for the He-filled to the airfilled cell as a function of the chopping frequency.
One notices that, both at high and low frequencies the experimental results agree very well with the predictions of our model. In the intermediate frequency range the PA signal is, of course, not given by the simple expressions of Eqs. (6) and (8) but rather by Eqs. (4) and (3). To make sure that the PA signal for the He-filled cell was correct, we have measured it as a function of time. This procedure was then repeated 3 times at each frequency, which assured us adequate reproducibility of our measurements. The reason for doing so is that, because of its lightness and smallness, helium has a high escaping rate from closed cells. A typical plot of the acoustic signal for the He-filled cell as a function of time is shown in Fig. 1. The ratio of the He-to-air signal is then calculated from these plots from its value at $t=0$.

Finally, as a further check of the present theory, we have measured the PA signal for Ge in the low-frequency range $(<25 \mathrm{~Hz})$ for different backings; those used were Teflon and brass. The observed signals were found to be independent of the material used. This result is again in strong support of the present theory and in contrast with $\mathrm{RG}$ predictions. The RG theory, for optically opaque and thermally thin samples, predicts that the PA signal should depend on the thermal properties of the backing as $\left(\alpha_{b}\right)^{1 / 2} / k_{b}$, whereas from our model [Eq. (6)] it should be independent of the backing material.

${ }^{1}$ A. Rosencwaig, Opt. Commun. 7, 305 (1973).

${ }^{2}$ A. Rosencwaig and A. Gersho, J. Appl. Phys. 47, 64 (1976).

${ }^{3}$ L. C. Aamodt, J. C. Murphy, and J. G. Parker, J. Appl. Phys. 48, 927 (1977).

${ }^{4}$ L. C. Aamodt and J. C. Murphy, J. Appl. Phys. $\underline{49}$, 3036 (1978).

${ }^{5}$ F. Alan McDonald and G. C. Wetsel, Jr., J. Appl. Phys. 49, 2313 (1978).

${ }^{6}$ H. S. Carslaw and J. C. Jaeger, Conduction of Heat in Solids (Clarendon, Oxford, 1959). 